\title{
La tutoría: escenario de aprendizaje en la Fundación Universitaria del Área Andina*
}

\author{
Nelly Yolanda Céspedes Guevara** \\ Juan David Adame Rodríguez ${ }^{* * *}$ \\ Johana Julieth Marín García***
}

Recibido: 10 de septiembre de 2019 • Aprobado: 13 de noviembre de 2019

\section{Resumen}

La investigación desarrollada se realiza a través de una caracterización global, en la que los escenarios nacionales y locales proporcionan un acercamiento al concepto de tutoría explorado desde el análisis de las hipótesis de progresión, que identifican el estado actual en el que se encuentra la Fundación Universitaria del Área Andina, en relación con los procesos de apoyo académico e integral a los estudiantes. La investigación se desarrolla desde el paradigma cualitativo, tomando como referente el estudio de caso múltiple, a través de la formulación de una hipótesis de progresión, que determina una de las formas de presentación de resultados. El análisis del contenido de las diferentes unidades de información, obtenidas a través de las categorías propuestas en la sistematización de las experiencias tutoriales de ciertas universidades, permitió identificar la tendencia a la que apunta el proceso tutorial a nivel global, así como determinar el estado actual en el que se encuentra la institución de educación superior en cuestión en este aspecto.

Palabras clave: aprendizaje, conocimiento científico, conocimiento profesional del profesor, enseñanza, formación profesional, tutoría.

Artículo de investigación científica y tecnológica. Este artículo de investigación fue producto de la investigación realizada en el marco de la convocatoria de proyectos de investigación del 2015 de la Dirección Nacional de Investigación de la Fundación Universitaria del Área Andina. Citar como: Céspedes Guevara, N. Y., Adame Rodríguez, J. D., \& Marín García, J. J. (2020). La tutoría: escenario de aprendizaje en la Fundación Universitaria del Área Andina. Análisis, 52(96), 203-220. doi: https://doi.org/10.15332/21459169/5314

* Doctorado en Educación. Fundación Universitaria del Área Andina. Investigador Junior. Líder del Grupo de Investigación de Ciencias Básicas Areandina. Contacto: ncespedes@,areandina.edu.co ORCID: https://orcid.org/0000-0003-3490-342X

** Candidato a Doctor en Educación. Fundación Universitaria del Área Andina. Investigador Grupo de Investigación de Ciencias Básicas Areandina. Contacto: juadame@areandina.edu.co ORCID: https://orcid.org/0000-0001-5626-0694

Médico Cirujano. Fundación Universitaria del Área Andina. Investigador Grupo de Investigación de Ciencias Básicas. Contacto: imarin5@areandina.edu.co, ORCID: https://orcid.org/0000-0002-7607-6648 


\title{
Tutoring: learning scenario at the Fundación Universitaria del Área Andina (University Foundation of the Andean Area)
}

\author{
Nelly Yolanda Céspedes Guevara \\ Juan David Adame Rodríguez \\ Johana Julieth Marín García
}

\section{Abstract}

The research developed is carried out through a global characterization, in which national and local scenarios provide an approach to the concept of tutoring explored from the analysis of the progression hypotheses, which identify the current state of the Fundación Universitaria del Area Andina, in relation to the processes of academic and comprehensive support for students. The research is developed from the qualitative paradigm, taking as a reference the multiple case study, through the formulation of a progression hypothesis, which determines one of the forms of presentation of results. The content analysis of the different units of information, obtained through the categories proposed in the systematization of the tutorial experiences of certain universities, allowed to identify the tendency to which the tutorial process points at a global level, as well as to determine the current state of the institution of higher education in this regard.

Keywords: learning, scientific knowledge, professional knowledge of the teacher, teaching, professional training, tutoring. 


\title{
L'accompagnement du tuteur : un espace d'apprentissage dans la Fundación Universitaria del Área Andina
}

\author{
Nelly Yolanda Céspedes Guevara \\ Juan David Adame Rodríguez \\ Johana Julieth Marín García
}

\section{Résumé}

Cette recherche a été développée à partir d'une caractérisation globale sur plusieurs contextes nationaux et locaux qui donnent un aperçu de l'accompagnement par un tuteur. Ce concept est ensuite analysé au travers des hypothèses de progression qui identifient l'état actuel de la Fundación Universitaria del Área Andina en ce qui concerne le soutien académique intégral aux élèves. Le paradigme assumé est qualitatif et, plus précisément, l'étude de cas multiple et la formulation d'une hypothèse de progression. L'analyse du contenu des différentes unités d'information, obtenues au travers des catégories proposées à partir de quelques universités, a permis d'identifier une tendance globale ainsi qu'à déterminer l'état actuel de la Fundación Universitaria del Área Andina.

Mots clés : apprentissage, connaissance scientifique, connaissance professionnelle $\mathrm{du}$ professeur, enseignement, formation professionnelle, tuteur. 


\section{Introducción}

El proceso tutorial es un espacio que genera una serie de herramientas pedagógicas a los docentes para el acercamiento del conocimiento a los estudiantes; en este sentido, una tutoría se puede asociar con un esquema de trabajo en torno a las reflexiones teóricas de una disciplina de formación específica vinculada a un área pedagógica, que constituye una perspectiva de estudio y referente para los estudiantes como apoyos externos al desarrollo cognitivo ofrecido en las estructuras cotidianas de clase.

Desde este punto de vista, la tutoría ofrece esquemas de producción de conocimiento, en los que no solo se trabaja con las carencias o falencias de los estudiantes, sino que a su vez se pueden potencializar los conocimientos de los estudiantes traídos de sus escenarios laborales con miras al perfeccionamiento desde la formalidad de los espacios de clase.

En este sentido, en el marco de la convocatoria de proyectos de investigación del 2015, en la Fundación Universitaria del Área Andina, se da la oportunidad de mostrar cómo ha sido este desarrollo al interior de los procesos académicos de la universidad.

De acuerdo con Lara (2002), la tutoría se establece como un punto de partida en la construcción de escenarios de conocimiento que permitan intervenir las prácticas educativas desde los docentes y los procesos de aprendizaje de los estudiantes. Esta relación se encuentra mediada por los propósitos e intenciones que se busquen en el nivel formativo; es decir, en el nivel de formación universitaria se debe contemplar la necesidad de vincular la tutoría al desarrollo de habilidades de pensamiento que se generan en los estudiantes como parte de su formación profesional.

Según Fernández y Escribano (2008):

La tutoría universitaria es considerada hoy en día como una herramienta de gran importancia en la formación universitaria. Si bien como profesores, muchas veces hemos aconsejado a nuestros alumnos sobre diversos aspectos de la tarea universitaria, ahora esa dedicación se ofrece como un importante rol universitario que ha sido institucionalizada y formalizada como un derecho. (p. 2)

Esto implica que el proceso tutorial debe ser entendido como un esquema de trabajo formalizado dentro de las instituciones de educación universitaria al servicio de los estudiantes, con el ánimo de generar procesos más completos, en donde el acercamiento al conocimiento sea biunívoco y proporcione relaciones de enseñanza-aprendizaje más fluidas para todos los actores educativos.

El proceso tutorial es un reto y una oportunidad a la que se enfrenta la Universidad del siglo xxI, en cuyo sentido una de las necesidades primordiales 
por las que surge el proyecto de investigación es dar alcance a los procesos educativos desde el acompañamiento de los docentes a los estudiantes en lo disciplinar, lo pedagógico y lo formativo.

Desde esta perspectiva, el desarrollo procesual de los estudiantes en el transcurso del ciclo universitario es vital para el crecimiento personal de cada individuo, y la universidad debe contribuir a mejorar los niveles de responsabilidad social que tiene con los estudiantes cuando ingresan a su vida profesional.

La pertinencia del proceso tutorial se puede evidenciar en la posibilidad de analizar los niveles de acompañamiento desde las diferentes disciplinas y carreras; así, la tutoría debe constituirse en el eje central de cada programa académico a través de la transversalización del concepto tutorial, asumiendo el reto de plantear estrategias que sean motivantes e incluyentes a una comunidad educativa.

\section{Antecedentes}

Dentro del espectro de los antecedentes en el trabajo tutorial se puede evidenciar el trabajo del gobierno del Perú en el fortalecimiento de un programa de apoyo tutorial titulado Marco conceptual de la tutoría y orientación educacional en la EBR (2015): “La tutoría que se inscribe en el campo de la orientación, constituye una estrategia o modalidad para abordarla en las instituciones educativas. En este sentido, toda tutoría es orientación, pero no toda orientación es tutoría" (p. 30). Dichos aspectos permiten identificar la relevancia que el proceso tutorial debe cobrar en los escenarios de enseñanza y aprendizaje de cualquier disciplina.

En este recorrido se encuentra el trabajo de la Universidad de Guadalajara, titulado "Una aproximación al concepto de tutoría académica en el Centro Universitario de Ciencias de la Salud" (Lara, 2002), en el que trabajo se realiza una aproximación teórico-práctica desde la recuperación del concepto tutorial y la adaptación realizada para el contexto particular de Ciencias de la Salud. Este trabajo muestra la importancia del escenario tutorial tanto en las prácticas educativas como en los distintos niveles educativos reflejados en el sistema de educación mexicano.

Las perspectivas teóricas del estudio de las tutorías se encuentran ubicadas desde el constructivismo social. Benarroch y Sanz (s. f.) proponen que el desarrollo del proceso tutorial se encuentra enmarcado en un esquema teórico, donde el aprendizaje se presenta en un desarrollo individual, en el que cada persona construye su acercamiento al conocimiento, a través de una serie de situaciones que hacen parte de la complejidad personal, pero que cuando se contextualizan se puede construir en colectivo.

Según Berranoch y Sanz (s. f.), "las cogniciones representan sistemas de relaciones organizados internamente que abarcan un conjunto de reglas para procesar información o conectar una serie de eventos en la experiencia personal. 
El pensamiento cognitivo es, pues, una relación activa, dinámica, de eventos" (p. 25), lo que implica que el desarrollo del pensamiento se visibiliza en las necesidades que cada individuo potencializa como ser individual y colectivo. Por lo tanto, el proceso tutorial se ve enmarcado en un desarrollo colectivo, en el que los esquemas de conocimiento se construyen desde lo personal, hacia lo colectivo, con el fin de establecer relaciones de trabajo biunívocas entre los estudiantes y los docentes que ejercen el rol de tutor.

En este marco de referencia y desde unos referentes teóricos, Berrancoh y Sanz (s. f.) sostienen lo siguiente:

El proyecto Tuning asigna al profesor el papel de ayudar al estudiante en el proceso de adquisición de competencias. El concepto de competencia abarca tres ámbitos paralelos: lo que el alumno va a ser capaz de conocer (área académica), hacer (habilidades y destrezas) y ser (actitudes y responsabilidades). Esto cambia la formulación de los objetivos educativos fijados tradicionalmente y mueve a rediseñar las actividades docente y discente. (p. 100)

Dicho escenario propende por un análisis tutorial ligado a los niveles de competencias como ejes centrales de la educación universitaria con formación en competitividad.

Según Amor (2012), "la orientación está presente en todas las etapas educativas, en unas de forma más explícita que en otras, pero quizás es en la etapa universitaria dónde menos manifiesta su necesidad" (p. 85). Esto implica que el proceso tutorial se puede considerar como el eje de trabajo de la comunidad educativa en aras del trabajo colectivo en la formación profesional.

Zabalsa (2006, citado por Amor, 2012) establece que la tutoría debe ser un apoyo a la inmadurez del alumno universitario, al considerar que comienzan sus estudios sin haber alcanzado la autonomía necesaria para desenvolverse de forma adecuada en la vida universitaria. Desde esta perspectiva, es necesario considerar las estructuras de trabajo de la investigación centradas en el reconocimiento del proceso de tutoría, desde el desempeño de los docentes y las caracterizaciones particulares de los estudiantes.

La interpretación y ejercicio de tutoría en la Fundación Universitaria del Área Andina (FUAA) se enmarca en la estrategia de prevención de la deserción que se adelanta desde el 2008, y busca tener una incidencia en los ejes individual, socioeconómico, académico, vocacional e institucional, con el fin de mitigar el impacto que posee este fenómeno al interior de las aulas. Dicha estrategia, en cabeza de Bienestar Institucional, responde a los modelos de seguimiento propuestos desde el Ministerio de Educación Nacional (2008) y hace mediciones con base en las variables planteadas en el software SPADIES, centradas en la identificación de materias con alta mortalidad académica y estudiantes en riesgo de deserción direccionan un trabajo sistemático orientado a la generación de impacto en la comunidad académica, cuyo pilar se constituye en la tutoría. 
En este sentido, la tutoría se fundamenta en un proceso de identificación y seguimiento de estudiantes en riesgo de deserción, gracias a un modelo de flujo de información entre los estamentos administrativos y académicos con una preponderante participación de las áreas transversales (Departamento de Ciencias Básicas y Departamento de Humanidades), en las que se define el rol de docente tutor quien, junto a los docentes de acompañamiento y la red integral de monitores, se constituye en asesor y agente observador del proceso, con el fin de brindar apoyo desde lo académico o remisión a otros estamentos que aporten a mitigar la deserción estudiantil. De este modo, el proyecto contempla la tutoría como el proceso de acompañamiento flexible y personalizado adicional a la carga académica, realizado por estudiantes y docentes tutores, cuyo fin principal es fortalecer conocimientos, resolver inquietudes y preparar al grupo de estudiantes inscritos, generando mejoramiento en su rendimiento académico mediante la integración de conocimientos y experiencias entre los participantes. Las dimensiones de las tutorías son afectivas, de conocimiento, de investigación, de orientación personal y de comunicación.

\section{Marco teórico}

Como parte del desarrollo de la construcción teórica del proyecto de investigación La tutoría como espacio académico en la FUAA, se presenta una estrategia de recopilación documental, en donde se inició la búsqueda de información a través de la web de los diferentes escenarios académicos, en los que el proceso tutorial ha cobrado una especial relevancia, siendo característica la necesidad de visualizar el entorno tutorial como un elemento esencial de un proceso de enseñanza y aprendizaje en las disciplinas del conocimiento. El grupo de investigación trabajó desde tres escenarios así: Europa, analizando las Universidades de Sorbona, Bolonia y Barcelona, a partir de las declaraciones europeas sobre el contexto tutorial y sus alcances a nivel académico; América Latina, a partir del Asociación Nacional de Universidades e Instituciones de Educación Superior de México y la Universidad de Buenos Aires de Argentina, que han sido los abanderados en el proceso tutorial y de acompañamiento a los estudiantes; Colombia, con las Universidades de Antioquia, La Sabana, Industrial de Santander y Nacional, que se han caracterizado por tener programas consolidados de trabajo tutorial permanente con seguimiento y alcances demostrables.

\section{La tutoría en el escenario internacional: Europa}

En el contexto de las universidades europeas se ha producido un incremento en el número de estudiantes que acceden a estudios superiores. Actualmente, la universidad se ha masificado por lo que la complejidad tipológica del estudiantado ha aumentado considerablemente $y$, con ella, las necesidades formativas y de desarrollo profesional. El trabajo del estudiante tiene como objetivo el logro de competencias académicas y profesionales que configuran su perfil de egreso 
al término de un determinado grado universitario: ello permite señalizar el perfil y contemplar los itinerarios de formación y desarrollo, así como sus conexiones con la oferta de posgrado con miras a su eventual especialización.

La finalidad de la tutoría puede ser vista como un proceso orientador que desarrollan de manera conjunta profesor y estudiante, en aspectos académicos, profesionales y personales, con le objeto de establecer un programa de trabajo que favorezca los escenarios de trabajo en torno al desarrollo y consecución de una carrera universitaria, a través de las distintas interacciones que existen entre los docentes y los estudiantes en la construcción de un esquema de conocimiento reflexivo sobre una determinada materia o proyecto interdisciplinar.

La tutoría universitaria de acuerdo con los modelos europeos puede entenderse y practicarse bajo tres aspectos:

- La tesis de la disociación se oferta como ayuda a la enseñanza presencial en la que, con un horario específico, tanto su asistencia como su participación son voluntarias, lo que implica que la participación en estos espacios no se constituye como un factor de aprobación en las asignaturas respectivas.

- La tesis de la complementariedad con la que las tareas, responsabilidades y estrategias de orientación, como las tutorías personalizadas o los seminarios grupales, se incorporan a la función docente como un complemento a la formación recibida en ámbitos relacionados con la madurez personal y la orientación profesional, favoreciendo el éxito académico en su trayectoria universitaria.

- La tesis de la integración cumple una función tutorial y docente integradora, enmarcada en la idea de la búsqueda de la excelencia académica y la consideración del individuo como un ser activo y proactivo de formación; a través de esta estragia, el desarrollo de los estudiantes se encuentra ligado a procesos de aprendizajes centrados en necesidades, intereses y competencias de los estudiantes a través de formación profesional.

Como apuntan la Obiols y Giner (2011):

Bolonia pone de manifiesto la importancia de la orientación como un elemento clave en la formación universitaria. La tutoría académica es la herramienta que permite realizar este proceso de orientación. Es un proceso de acompañamiento de carácter formativo, orientador e integral desarrollado por docentes universitarios. Su finalidad es la de facilitar a los alumnos todas las herramientas y toda la ayuda necesaria para alcanzar con éxito tanto los hitos académicos como personales y profesionales que la universidad les plantea. (p. 20)

Ello implica que la tutoría no es solo un espacio donde resolver dudas, sino que se constituye en una reunión de trabajo entre profesor y alumno o grupo reducido de alumnado. 


\section{La tutoría en el escenario nacional}

La tutoría en la educación superior latinoamericana, y especialmente en Colombia, se encuentra atravesando un periodo de trascendental importancia. El crecimiento vertiginoso de nuestra población urbana ha traído consigo un incremento multitudinario en la búsqueda de acceso a la educación superior, a la que se ha tratado de dar respuesta, no siempre con criterios de eficiencia y calidad. De acuerdo con lo expuesto por Aldana (2001), la masificación de la matrícula universitaria en Colombia se multiplicó 80 veces entre 1950 y el final del siglo, y el número de programas de pregrado, que en 1960 era de 190, en 1999 es de aproximadamente 8.000 (Ariza \& Ocampo, 2005).

La tutoría como intervención formativa destinada al seguimiento académico de los estudiantes es una estrategia pedagógica que vienen desarrollando diferentes universidades, particularmente algunas de Colombia, como una manera de brindar respuesta a la problemática de deserción. Esta situación llevó al Ministerio de Educación a extender una convocatoria, para diseñar una herramienta y una metodología de seguimiento al rendimiento académico universitario, para lo cual se realizó una investigación en la que participaron 70 universidades, el Instituto Colombiano para la Evaluación de la Educación (Icfes) y el Instituto Colombiano de Crédito Educativo y Estudios Técnicos en el Exterior (Icetex). Los resultados indicaron la importancia de implementar estrategias académicas de apoyo; una de ellas es la tutoría, en especial durante los primeros semestres, en los que se presenta mayor deserción, debido, entre otras causas, a la falta de adaptación a un nuevo sistema educativo (Cardozo-Ortiz, 2011).

Tal vez el documento más representativo que representa esta tendencia es el denominado Plan de Acción de Turín, suscrito por Colombia en el 2000. Este documento establece, por ejemplo, el requerimiento de que las universidades latinoamericanas diseñen currículos "más flexibles (con sistemas de crédito y opciones alternativas) y organizados en programas secuenciales más cortos, con la posibilidad de acceder al mercado de trabajo al completar el primer nivel" (Ariza \& Ocampo, 2005, p. 105).

En relación con décadas anteriores, el concepto de tutor ha cambiado significativamente: el tutor es un docente que tiene un saber propio para enseñar y que, a través de distintas estrategias, metodologías y opciones pedagógicas, diseña materiales, cursos, aulas virtuales, etc., para socializar el conocimiento; de igual manera, realiza asesorías (epistolares, telefónicas, presenciales, por chat, foros, e-mail, etc.) para acompañar los procesos educativos. En Colombia, el desarrollo de este concepto no ha tenido una definición y una trayectoria significativa, al punto de que todo lo que se dice de él es prestado y acomodado a las filosofías de los proyectos educativos. Carecemos de una reflexión sería en torno a la tutoría, al tutor, al significado de su concepto, a su perfil y, por ende, a su formación (González, 2005). 
En Colombia, el desarrollo de la tutoría más conocido es el de los programas de educación superior a distancia, donde el tutor es el intermediario entre el autoaprendizaje del estudiante y los módulos elaborados para el desarrollo de competencias tecnológicas o profesionales en un saber específico. El tutor, a través de estrategias didácticas complementarias, asesora al estudiante en su proceso de aprendizaje autónomo (Sánchez, 2006; Cardozo-Ortiz, 2011).

Si bien esta labor de acompañamiento tutorial universitario en Colombia ha tenido un principal arraigo en la figura del profesor, existen experiencias significativas de tutoría entre pares, en las que se visibiliza el papel del estudiante como actor principal en el logro de soluciones efectivas ante dificultades como el bajo rendimiento académico. Esto ha permitido comprender que existen otras formas de acercarse a las realidades de los estudiantes, para lograr que sean ellos mismos los que aporten en la construcción de mejores condiciones de vida universitaria (Cardozo-Ortiz, 2011).

Esta situación se constituye en uno de los retos más decisivos para la institución universitaria que debe tener en cuenta fenómenos macroeconómicos vinculados con la globalización y la "simbiosis de la ciencia y la tecnología", de acuerdo con lo expuesto por Aldana (2001). Además, Ariza y Ocampo (2005) hace énfasis en lo siguiente:

Este progreso produce cambios en la teoría y la práctica de la enseñanza y el aprendizaje, en los canales utilizados para la comunicación entre alumnos y profesores, en las estructuras administrativas y académicas de las universidades y en las relaciones de trabajo entre los docentes y las instituciones. (p. 50)

La primera función de la tutoría es la de una pedagogía del acompañamiento, inscrita como situación didáctica intermedia entre la clase magistral y la entrevista individual o clase particular, con el objeto de contribuir en la búsqueda de la verdad científica y del mejoramiento de los procesos académicos. La segunda es una función instructiva para profundizar en las destrezas y conocimientos en un determinado campo del saber. Otra función es la de motivación y estímulo a la formación de actitudes intelectuales y éticas hacia la ciencia y la responsabilidad social (Sánchez, 2006).

Para terminar, tanto el documento de la Unesco y del Banco Mundial como la Declaración de Bolonia invitan a reflexionar de manera efectiva y positiva sobre la importancia y significado de la tutoría universitaria, que contribuye a preparar al futuro profesional para que intervenga en la resolución de los problemas no solo laborales y propios de la disciplina, sino en los de carácter social. He aquí el reto que debe asumir el docente tutor, pues se trata de la máxima responsabilidad gracias a la cual coadyuva en la formación de personas idóneas para construir una nueva sociedad con visión prospectiva (Calle \& Saavedra, 2009).

El docente universitario convencional, dedicado principalmente a la transmisión de un conocimiento, debe tender a convertirse en un tutor, acompañante en el 
proceso del aprender. El docente debe convertirse en tutor, en un proceso en donde los estudiantes buscan adquirir y los tutores ayudan a adquirir; los estudiantes aprenden haciendo y sus instructores funcionan más como tutores que como profesores. Por lo tanto, una de las funciones del docente es convertirse en guía y modelo de sus alumnos. El acompañamiento tutorial no solo debe tener en cuenta el cumplimiento de un programa académico, sino que debe crear en el estudiante el entusiasmo y la pasión por el proceso de aprendizaje (Ariza \& Ocampo, 2005).

\section{La tutoría en la Fundación Universitaria del Área Andina}

La prevención es el enfoque principal de la tutoría en la FUAA, sin dejar desdeñar las intervenciones frente a problemáticas ya dadas. Las tutorías no tienen ningún costo para el estudiante, no afectan el desempeño académico en cuanto a notas se refiere y los horarios se ajustan de acuerdo con la disponibilidad de los estudiantes y de los docentes tutores, quienes realizan la labor teniendo en cuenta la distribución del tiempo establecido para tal fin, según lo dispuesto en la distribución de la carga docente, por lo que no tiene remuneración económica adicional y se llevan registros mensuales de asistencia a tutorías académicas.

La experiencia del Departamento de Ciencias Básicas de la fuAA ha evidenciado que las tutorías generan una mejoría entre el primer parcial y el examen final en una asignatura dada, encontrándose entonces que un alto porcentaje de estudiantes logran culminar exitosamente la formación en los semestres previstos para cada programa académico, lo que representa una alta efectividad del servicio.

En el marco del Proyecto G, liderado desde el Ministerio de Educación de la Republica de Colombia, se da inicio en el 2011 al Programa Integral de Permanencia Estudiantil (PIPE) en la FUAA, cuyo objetivo se centró en el desarrollo de estrategias para mitigar la deserción estudiantil que, para dicho periodo, se ubicaba en el $15,7 \%$. La fase exploratoria de este proyecto inicia con la identificación de alertas de deserción estudiantil tales como bajo rendimiento académico o determinantes individuales y socioeconómicos; en este sentido, se articulan agentes observadores desde distintos ámbitos institucionales que permitan la identificación de casos y su posterior derivación hacia los recursos de que dispone la institución para el apoyo en la resolución de la condición particular.

Para la articulación del PIPE se incorpora un equipo de docentes de acompañamiento al estudiante, quienes pertenecen a cada programa académico de pregrado en su modalidad presencial. El docente acompañante, en articulación con un grupo de estudiantes que componen un sistema integral de monitores, tienen como objetivo identificar y evidenciar casos en riesgo a la coordinación del proyecto para asesoría y seguimiento. Este equipo interactúa con los tutores del 
Departamento de Ciencias Básicas, quienes en su condición de área transversal aportan las herramientas académicas y el acompañamiento que fortalezcan la adquisición de competencias específicas. Por su parte, la red de monitores se compone de tres principales figuras: 1) los monitores de aula (MAU), quienes identifican la inasistencia a clase; 2) los monitores académicos (MAC), quienes tienen como principal función promover grupos de estudio y aprovechamiento de espacios tutoriales institucionales, y 3) los monitores asistentes (MAS), quienes tienen la función de asistir a cada docente acompañante para las actividades del PIPE en los programas académicos. Los monitores mencionados hacen parte de la comunidad estudiantil y son seleccionados por sus cualidades de liderazgo en su grupo, además de su buen desempeño académico, constituyéndose en pares vinculadores entre sus compañeros en dificultades de distinta índole.

Por otra parte, la figura del docente acompañante se circunscribe en la recepción de casos en riesgo y la remisión al área encargada, siendo este el ente articulador entre la red de monitores y la Oficina de Permanencia. En este esquema, la figura del docente tutor está sujeta a la resolución de dificultades académicas de estudiantes remitidos desde distintos integrantes de la red, pero también al acompañamiento estructurado de manera adicional y complementaria al trabajo en el aula.

\section{Metodología}

Se optó por un diseño orientado desde el paradigma cualitativo-interpretativo, teniendo en cuenta que la investigación es de carácter educativo. Como el propósito del proyecto es contrastar el estado actual del proceso tutorial en educación superior a nivel mundial con respecto al proceso que adelanta la fuAA con dicho referente, se aplicó el estudio de caso como estrategia metodológica, con el fin de establecer un rigor científico de validación a la investigación. Por otra parte, se propone para este proyecto el análisis de mecanismos para el análisis de la información obtenida a partir de los diferentes documentos institucionales, grabaciones, entrevistas estructuradas, diario de campo y test.

\section{Resultados y discusión}

En el contexto de las múltiples funciones que asume la universidad contemporánea, hay quienes reclaman un papel de la universidad más comprometido con la sociedad a la que pertenece, promoviendo el análisis crítico de la realidad y transformándola a través de una visión de multiplicidad, más aun en el escenario de la universidad pública, de la que se espera pertinencia en términos de su impacto social y, de manera indisoluble, calidad, en la medida en que ayuda a la integración social. Es así como el reto está en la inserción de la educación superior en un mundo globalizado sin dejarse absorber por lo concerniente al 
mercado, priorizando la generación de nuevo conocimiento en medio de la discusión, aunque esto genere más incertidumbre; surge así un concepto de educación de calidad como aquella que promueve en sus estudiantes el alcance de logros intelectuales, sociales y emocionales acudiendo a su contexto socioeconómico y situación familiar.

En términos prácticos, la propuesta educativa se plasma en la malla curricular y en su enfoque específico que debe responder a las necesidades, intereses y problemas de los que asisten a la universidad, y esta función debe ser eficiente desde lo cultural y lo administrativo. En últimas, la educación es repensada como la capacitación y la formación para la convivencia, así como para la distribución equitativa del conocimiento y uso de nuevas tecnologías, convirtiéndose en un eje estratégico para el desarrollo de los países en crecimiento.

Dicho escenario no nos asiste en la realidad colombiana, en la que las universidades no corresponden ni cualitativa ni cuantitativamente a las necesidades del país. Se evidencia así un fracaso de la universidad tradicional por su incapacidad de incorporar el ámbito intelectual y el mundo productivo. En pos de corregir dicha situación, les corresponde a las universidades acompañar a sus estudiantes más allá del aula de clase, y es aquí donde adquiere importancia la tutoría como estrategia para garantizar la calidad, priorizando el aprendizaje sobre la enseñanza.

En este marco, la Universidad de Antioquia, como institución de educación superior reconocida por sus proyectos e impacto a nivel científico, académico y social, asume dentro de sus objetivos estratégicos el mejoramiento de los programas de formación y capacitación usando como iniciativa el programa de promoción de la permanencia estudiantil. De allí surge el "Proyecto para el Fortalecimiento del Programa Institucional de Permanencia 2012-2013 de la Universidad de Antioquia", liderado por la Vicerrectoría de Docencia de la Universidad, y cuyo propósito es propiciar la formación integral, así como colaborar en la orientación vocacional y profesional. De manera coherente, se plantea en su estatuto profesoral que, dentro de las actividades de los docentes, figura la tutoría académica a estudiantes, cuyo sentido es ser orientador y guía durante el tránsito del estudiante por la universidad. Las diferentes iniciativas de programas tutoriales en la Universidad de Antioquia son validadas no tanto por su complejidad administrativa, sino más bien por su intención e impacto en el devenir de cada estudiante y sus necesidades.

En última instancia, la Universidad de Antioquia contempla la tutoría como un conjunto de estrategias que atienden a las particularidades de cada programa y que adopta los diferentes planteamientos desde lo administrativo, lo particular y lo espontáneo, porque al final prioriza la atención oportuna e integral a sus estudiantes. Allí surge la tutoría integral con el deseo principal de satisfacer no solo las necesidades de formación de alta calidad, sino también las expectativas sociales, promoviendo un seguimiento del desarrollo intelectual, moral y social, acompañado en su diario discurrir. 
En cuanto a la FUAA, el presente estudio nos permite comprender que posee un programa de tutorías en vías de consolidación, que prioriza sus intervenciones en términos de mitigación de la deserción estudiantil y, de esta manera, se centra en los primeros tres semestres, estrategia exitosa por cuanto los indicadores de permanencia son favorables; no obstante, la intención de la institución plantea la necesidad de apuntar a la educación con cobertura y calidad, con lo cual se plantea un reto desde la dirección, coordinación y gestión administrativa y académica hacia la implementación de programas que favorezcan el acompañamiento integral a sus estudiantes durante toda su carrera universitaria.

Es imperiosa la migración de un modelo tutorial de transmisión frugal de conocimientos para la no pérdida académica hacia la construcción de escenarios de conversación que permitan identificar el contexto y generar conocimiento formativo y pertinente. De este modo, nos permitimos hacer un llamado a la evolución del programa hacia un proceso tutorial de alta calidad, logro que pasa por la selección y promoción de docentes con capacidad de conectarse con el campo experiencial del estudiante, así como con el interés genuino por conocer sus fortalezas, intereses y dificultades de aprendizaje. Se debe propender por una política institucional que permita tejer relaciones humanas significativas en las que toda la comunidad se sienta comprometida y dispuesta con sus ideales personales y profesionales.

A continuación, se describirá en detalle cada una de las categorías de organización que son objeto de análisis, tomando como referente el trabajo de Martínez, Valbuena y Andrade (2013), que se modificó de forma contextualizada conforme a las características propias que subyacen en esta investigación:

- Contenidos tutoriales: corresponde a los tipos de contenidos que el docente tutor enseña desde su proceso de orientación al estudiante, tales como conceptos, procedimientos, actitudes y valores. Esto implica abordar la manera como están constituidos dichos contenidos.

- Fuentes y criterios de selección de contenidos tutoriales: hace referencia a las fuentes que utiliza el docente tutor para seleccionar los contenidos desde su proceso de orientación estudiantil, tales como experiencias personales, materiales escritos (textos escolares, lineamientos curriculares, documentos institucionales, etc.), saberes de personas, entre otros. Asimismo, esta categoría incluye los criterios que utiliza el profesor para seleccionar dichos contenidos.

- Referentes epistemológicos del conocimiento tutorial: corresponden a la naturaleza de los diferentes tipos de conocimiento que intervienen en el proceso de orientación tutorial; por ejemplo, conocimiento de origen científico, conocimiento curricular, concepciones de los estudiantes y creencias populares entre otros.

- Criterios de validez del conocimiento tutorial: hace referencia a los principios y sujetos que determinan si el conocimiento que se produce en el proceso de orientación tutorial es legítimo. 


\section{Niveles de progresión propuestos para analizar y categorizar del conocimiento profesional de la tutoría de la FUAA}

Con el fin de caracterizar el conocimiento profesional que se tiene sobre la tutoría vista desde una perspectiva global y contemporánea, se identificarán diferentes unidades de información proveniente desde la sistematización de diversas experiencias exitosas en instituciones educativas de educación superior a nivel nacional e internacional, que a partir de la contratación con los siguientes niveles de progresión serán analizados a través de una matriz $H d P$.

\section{1a. Tutoría técnica (тT)}

Esta tutoría también se conoce como asesoría académica, en la que el estudiante solicita la colaboración de un docente con cierta experticia en determinada área del conocimiento, con el fin de conseguir una explicación o solucionar inquietudes sobre una temática específica. Se fundamenta desde lo conceptual y lo procedimental, pudiendo ser desempeñada por docentes que tienen grupos de estudiantes a su cargo, como de los que no.

\section{1 b. Tutoría monitoreada por asignación y asistencia (TMAA)}

En este modelo, el tutor es un estudiante aventajado (académicamente), que se presenta ante un par o un grupo de pares que presentan dificultades para desarrollar tareas (actividades académicas) o temáticas específicas en el campo del conocimiento. Se presenta como una variante de la tutoría técnica.

\section{Tutoría orientada desde la praxis (TOP)}

Los tutores son los responsables del seguimiento de prácticas que coadyuvan a la formación y desarrollo profesional de los estudiantes, buscando el perfeccionamiento de habilidades y competencias específicas, a través de la confrontación de experiencias vivenciales con referentes teórico-conceptuales adquiridos desde su formación. Algunos ejemplos de este tipo de acompañamiento se pueden evidenciar en prácticas clínicas, laboratorios, prácticas artísticas, instrumentales, deportivas, de corte empresarial y algunos tipos de pasantías que requieran de esta orientación.

\section{Tutoría integral formativa (TIF)}

También conocida como tutoría personalizada, en esta modalidad el profesor o tutor pretende conocer la situación específica de un estudiante o de un número 
determinado de estudiantes, en donde les orienta de forma personal sobre la planificación y ejecución de sus actividades (en casos específicos él le remite al órgano institucional competente para su acompañamiento). Uno de los puntos positivos de este tipo de tutoría es trabajar la autoestima de los estudiantes, que favorece el empoderamiento de sus responsabilidades y le permite generar nuevos retos. Esta tutoría supone un compromiso más profundo tanto por parte del tutor como por parte del estudiante, ya que abarca temáticas de índole intelectual, afectiva, social, académica, profesional, institucional, etc., siempre vistas desde la perspectiva de diversidad.

\section{Reflexiones finales}

Dado que las habilidades matemáticas son un requisito esencial para las ciencias e ingeniería, los estudiantes que llegan a una institución de educación superior y que carecen de estas habilidades se enfrentan a un obstáculo importante para una exitosa conclusión de sus estudios. Por eso, las tutorías son estrategias de acompañamiento académico a estudiantes que pertenecen a grupos vulnerables, con el fin de perfeccionar sus métodos de trabajo, desarrollar sus competencias cognitivas e instrumentales, y responder a las necesidades planteadas por las disciplinas cursadas para poder continuar sin mayores tropiezos sus estudios. En general, el valor y la importancia del proyecto de tutorías radican en el hecho de que está orientado a disminuir la deserción estudiantil, a través del refuerzo de los conocimientos en matemáticas, una de las materias que mayores problemas presenta para los estudiantes y que, en muchos casos, es la causante de la salida de las universidades. Además, las tutorías ayudan a enfrentar de mejor manera los ambientes de competitividad y presión frecuentes en las universidades. El proyecto de tutorías está integrado básicamente por los siguientes componentes:

- Una comunidad virtual conformada por profesores y estudiantes de colegio y universidad, quienes se comunican sobre temas relacionados con la matemática y la educación matemática haciendo uso de una plataforma en Internet.

- Un repositorio en línea con materiales para la enseñanza y aprendizaje de matemática (notas electrónicas disponibles libremente, libros de texto, ejercicios, exámenes y enlaces a sitios web de interés).

- Creación de un programa de tutoría en matemática para estudiantes de primer año de las instituciones de educación superior.

- Tutores que deben contar con un manejo de las herramientas digitales y programas específicos necesarios para realizar las tutorías tanto presenciales como virtuales, lo que implica tener manejo de plataformas de aprendizaje virtual.

- La Wiki, que se enfoca en errores frecuentes y malentendidos comunes de los estudiantes que se observan durante las sesiones de tutoría. 
- Un foro que permite a los coordinadores, tutores, profesores y todos los que están involucrados en el programa de mentores o interesados en su aplicación discutir cuestiones didácticas, dar su opinión sobre problemas y compartir las experiencias de los estudiantes

Si bien no se puede garantizar que el estudiante permanezca en la universidad, se le ofrecen las herramientas que están al alcance de la institución tanto de manera presencial como virtual, para que tenga un acompañamiento permanente y oportuno.

\section{Referencias}

Aldana, V. (2001). Tendencias, retos y mitos de la educación superior en Colombia. Congreso: Educación superior, desafío global y respuesta nacional. Bogotá: Universidad de los Andes.

Amor, M. (2012) La orientación y la tutoría universitaria como elementos para la calidad y la innovación en la Educación Superior. Modelo de Acción Tutorial (Tesis Doctoral). Universidad de Córdoba, Córdoba, España.

Ariza, G., \& Ocampo, H. (2005). El acompañamiento tutorial como estrategia de la formación personal y profesional: un estudio basado en la experiencia en una institución de educación superior. Universitas Psychologica, 4(1), 31-41. Recuperado de https://www.redalyc.org/articulo.oa?id=64740104

Berranoch, M., \& Sanz, M. (s. f.). Orientación y Tutoría Universitaria. Granada: Universidad de Granada.

Calle, M., \& R, Saavedra. (2009). La tutoría como mediación para el desarrollo autónomo del estudiante. Tabula Raza, 11, 309 -328.

Cardozo-Ortiz, C. (2005). Tutoría entre pares como una estrategia pedagógica universitaria. Educación y Educadores, 14(2), 309-325.

Fernández, G., \& Escribano, M. (2008). Las tutorías en la formación académica y humana de los alumnos en la Universidad de San Pablo ceU. xVI Jornadas Asepuma - IV Encuentro Internacional, 16(1), 605.

Gobierno del Perú. (2015.). Marco Conceptual de la Tutoría y Orientación Educacional en la EBR. Recuperado de https://www.academia.edu/5829303/Marco Conceptual de la Tutor\%C3\%ADa y Orientaci\%C3\%B3n Educacional en la EBR

González, E. (2005). La tutoría en la Universidad Colombiana: etapas, procesos y reflexiones. Revista Historia de la Educación Latinoamericana, 7, 241-258. 
Lara, B. (2002). Una aproximación al concepto de tutoría académica en el Centro Universitario de Ciencias de la Salud. Investigación en Salud, 4(1), s. p. Recuperado de http://www.redalyc.org/articulo.oa?id=14240106

Martínez, C., Valbuena, E., \& Andrade, A. (2013). El conocimiento profesional que los profesoresde ciencias de primaria tienen sobre el conocimiento escolar, en el Distrito Capital: un problema de investigación. En C. Martínez \& E. Valbuena (Comp.), El conocimiento profesional de los profesores de ciencias sobre el conocimiento escolar: resultados de investigación (pp. 13-34). Bogotá: Universidad Distrital Francisco José de Caldas. Bogotá.

Ministerio de Educación Nacional. (2008). Directiva ministerial No. 20, 29 de octubre de 2008

Sanchéz, M. (2006). La tutoría académica. Cuadernos de Psicopedagogía. Recuperado de http://www.uptc.edu.co/facultades/f_educacion/pregrado/ psicopedagogia/documentos/cuaderno 2-Tutoria.pdf

Obiols, M., \& Giner, A. (2011). El modelo educativo de Bolonia y competencias docentes. Aportaciones desde el coaching educativo. Girona: Universdiad de Girona. Recuperado de https://dugi-doc.udg.edu/bitstream/handle/10256/3726/321. pdf? sequence $=1 \&$ isAllowed $=\mathrm{y}$ 